\title{
Baryons, their interactions and the chiral symmetry of QCD
}

\author{
L. Ya. Glozman ${ }^{\mathrm{a}}$
}

${ }^{a}$ Institute for Theoretical Physics, University of Graz, Universitätsplatz 5, A-8010 Graz, Austria

An implication of the spontaneous chiral symmetry breaking in QCD is that at low energy and resolution there appear quasiparticles - constituent quarks and Goldstone bosons. Thus, light and strange baryons should be considered as systems of three constituent quarks with confining interaction and a chiral interaction that is mediated by Goldstone bosons between the constituent quarks. We show how the flavor-spin structure and sign of the short-range part of the Goldstone boson exchange interaction reduces the $S U(6)_{F S}$ symmetry down to $S U(3)_{F} \times S U(2)_{S}$, induces hyperfine splittings and provides correct ordering of the lowest states with positive and negative parity. We present a unified description of light and strange baryon spectra calculated in a semirelativistic framework. It is demonstrated that the same short-range part of Goldstone boson exchange also induces strong short-range repulsion in $N N$ system when the latter is treated as $6 Q$ system. Thus all main ingredients of $N N$ interaction are implied by the chiral constituent quark model since the long- and intermediate-range attraction appears in the present framework due to pion and correlated two-pion exchanges between quarks belonging to different nucleons. We also find a very strong short-range repulsion in $\Lambda \Lambda$ system with $J^{P}=0^{+}$. It then suggests that the compact $H$-particle should not exist.

\section{Spontaneous Chiral Symmetry Breaking and its Implications for Low-Energy QCD}

The QCD Lagrangian with three light flavors has a global symmetry

$S U(3)_{\mathrm{L}} \times S U(3)_{\mathrm{R}} \times U(1)_{\mathrm{V}} \times U(1)_{\mathrm{A}}$.

At low temperatures and densities the $S U(3)_{\mathrm{L}} \times S U(3)_{\mathrm{R}}$ chiral symmetry is spontaneously broken down to $S U(3)_{\mathrm{V}}$ in the QCD vacuum, i.e., realized in the hidden Nambu-Goldstone mode. A direct evidence for the spontaneously broken chiral symmetry is a nonzero value of the quark condensates for the light flavors $\left\langle|\bar{q} q|>\approx-(240-250 \mathrm{MeV})^{3}\right.$, which represent the order parameter. That this is indeed so, we know from three independent sources: current algebra, QCD sum rules, and lattice gauge calculations. There are two important generic consequences of the spontaneous chiral symmetry breaking. The first one is an appearance of the octet of pseudoscalar mesons of low mass, $\pi, \mathrm{K}, \eta$, which represent the associated approximate Goldstone bosons. The second one is that valence quarks acquire a dynamical or constituent mass. Both these consequences of the spontaneous chiral symmetry breaking are well illustrated by, e.g. the $\sigma$-model [1] or the Nambu and 
Jona-Lasinio model [2]. We cannot say at the moment for sure what the microscopical reason for spontaneous chiral symmetry breaking in the QCD vacuum is. It was suggested that this occurs when quarks propagate through instantons in the QCD vacuum [3].

For the low-energy baryon properties it is only essential that beyond the spontaneous chiral symmetry breaking scale (i.e. at low resolution) new dynamical degrees of freedom appear - constituent quarks and chiral fields which couple together 田5]. The low-energy baryon properties are mainly determined by these dynamical degrees of freedom and the confining interaction.

There is a very good analogy in solid state physics. For instance, the fundamental degrees of freedom in crystals are ions in the lattice, electrons and the electromagnetic field. Nevertheless, in order to understand electric conductivity, heat capacity, etc. we instead work with "heavy electrons" with dynamical mass, phonons and their interaction. In this case a complicated electromagnetic interaction of the electrons with the ions in the lattice is "hidden" in the dynamical mass of the electron and the interactions among ions in the lattice are eventually responsible for the collective excitations of the lattice phonons, which are Goldstone bosons of the spontaneously broken translational invariance in the lattice of ions. As a result, the theory becomes rather simple - only the electron and phonon degrees of freedom and electron-phonon interactions are essential for all the properties of crystals mentioned above.

We have recently suggested [6] that in the low-energy regime light and strange baryons should be considered as a system of 3 constituent quarks with an effective $Q-Q$ interaction that is formed of central confining part and a chiral interaction mediated by the Goldstone bosons between constituent quarks. This physical picture allows to understand a structure of the baryon spectrum and to solve, in particular, the long-standing problem of ordering of the lowest baryons with positive- and negative-parity.

\section{The Goldstone Boson Exchange Interaction and the Structure of Light and Strange Baryon Spectra}

The coupling of the constituent quarks and the pseudoscalar Goldstone bosons will (in the $S U(3)_{\mathrm{F}}$ symmetric approximation) have the form $i g \bar{\psi} \gamma_{5} \vec{\lambda}^{\mathrm{F}} \cdot \vec{\phi} \psi$ (or $g /(2 m) \bar{\psi} \gamma_{\mu} \gamma_{5} \vec{\lambda}^{\mathrm{F}}$. $\left.\psi \partial^{\mu} \vec{\phi}\right)$. A coupling of this form, in a nonrelativistic reduction for the constituent quark

spinors, will - to lowest order - give rise the $\sim \vec{\sigma} \cdot \vec{q} \vec{\lambda}_{i}^{\mathrm{F}}$ structure of the meson-quark vertex, where $\vec{q}$ is meson momentum. Thus, the structure of the $Q-Q$ potential in momentum representation is

$V(\vec{q}) \sim \vec{\sigma}_{i} \cdot \vec{q} \sigma_{j} \cdot \vec{q} \vec{\lambda}_{i}^{\mathrm{F}} \cdot \vec{\lambda}_{j}^{\mathrm{F}} D\left(q^{2}\right) F\left(q^{2}\right)$

where $D\left(q^{2}\right)$ is dressed Green function for chiral field which includes both nonlinear terms of chiral Lagrangian and fermion loops, $F\left(q^{2}\right)$ is meson-quark formfactor which takes into account internal structure of quasiparticles. At big distances $(\vec{q} \rightarrow 0), D\left(q^{2}\right) \rightarrow D_{0}\left(\vec{q}^{2}\right)=$ $-\left(\vec{q}^{2}+\mu^{2}\right)^{-1}$, where $D_{0}\left(\vec{q}^{2}\right)$ is free Klein-Gordon Green function in static approximation. Thus, $D(\vec{q}=0) \neq \infty$. It then follows from (2) that $V(\vec{q}=0)=0$, which is equivalent to $\int d \vec{r} V(\vec{r})=0$. Since at big interquark separations the spin-spin component of the 
pseudoscalar-exchange interaction is

$V\left(r_{i j}\right) \sim \vec{\sigma}_{i} \cdot \vec{\sigma}_{j} \vec{\lambda}_{i}^{\mathrm{F}} \cdot \vec{\lambda}_{j}^{\mathrm{F}} \mu^{2} \frac{e^{-\mu r_{i j}}}{r_{i j}}$

it then follows from the volume integral constraint that at short interquark separations the spin-spin interaction should be opposite in sign as compared to the Yukawa tail and very strong. It is this short-range part of the Goldstone boson exchange (GBE) interaction between constituent quarks that is of crucial importance for baryons: it has a sign appropriate to reproduce the level splittings and dominates over the Yukawa tail towards short distances. In a oversimplified consideration with a free Klein-Gordon Green function instead of the dressed one in (2) and with $F\left(q^{2}\right)=1$, one obtains the following spin-spin component of $Q-Q$ interaction:

$V\left(r_{i j}\right)=\frac{g^{2}}{4 \pi} \frac{1}{3} \frac{1}{4 m_{i} m_{j}} \vec{\sigma}_{i} \cdot \vec{\sigma}_{j} \vec{\lambda}_{i}^{\mathrm{F}} \cdot \vec{\lambda}_{j}^{\mathrm{F}}\left\{\mu^{2} \frac{e^{-\mu r_{i j}}}{r_{i j}}-4 \pi \delta\left(\vec{r}_{i j}\right)\right\}$.

Consider first, for the purposes of illustration, a schematic model which neglects the radial dependence of the potential function $V\left(r_{i j}\right)$ in (田), and assume a harmonic confinement among quarks as well as $m_{\mathrm{u}}=m_{\mathrm{d}}=m_{\mathrm{s}}$. In this model

$H_{\chi}=-\sum_{i<j} C_{\chi} \vec{\lambda}_{i}^{\mathrm{F}} \cdot \vec{\lambda}_{j}^{\mathrm{F}} \vec{\sigma}_{i} \cdot \vec{\sigma}_{j}$

If the only interaction between the quarks were the flavor- and spin-independent harmonic confining interaction, the baryon spectrum would be organized in multiplets of the symmetry group $S U(6)_{\mathrm{FS}} \times U(6)_{\text {conf }}$. In this case the baryon masses would be determined solely by the orbital structure, and the spectrum would be organized in an alternative sequence of positive and negative parity states. The Hamiltonian (5), within a first order perturbation theory, reduces the $S U(6)_{\mathrm{FS}} \times U(6)_{\text {conf }}$ symmetry down to $S U(3)_{\mathrm{F}} \times S U(2)_{\mathrm{S}} \times U(6)_{\text {conf }}$, which automatically implies a splitting between the octet and decuplet baryons.

The two-quark matrix elements of the interaction (5) are:

$$
\begin{gathered}
\quad<\left[f_{i j}\right]_{\mathrm{F}} \times\left[f_{i j}\right]_{\mathrm{S}}:\left[f_{i j}\right]_{\mathrm{FS}}\left|-C_{\chi} \vec{\lambda}_{i}^{\mathrm{F}} \cdot \vec{\lambda}_{j}^{\mathrm{F}} \vec{\sigma}_{i} \cdot \vec{\sigma}_{j}\right|\left[f_{i j}\right]_{\mathrm{F}} \times\left[f_{i j}\right]_{\mathrm{S}}:\left[f_{i j}\right]_{\mathrm{FS}}> \\
=\left\{\begin{aligned}
-\frac{4}{3} C_{\chi} & {[2]_{\mathrm{F}},[2]_{\mathrm{S}}:[2]_{\mathrm{FS}} } \\
-8 C_{\chi} & {[11]_{\mathrm{F}},[11]_{\mathrm{S}}:[2]_{\mathrm{FS}} } \\
4 C_{\chi} & {[2]_{\mathrm{F}},[11]_{\mathrm{S}}:[11]_{\mathrm{FS}} } \\
\frac{8}{3} C_{\chi} & {[11]_{\mathrm{F}},[2]_{\mathrm{S}}:[11]_{\mathrm{FS}} }
\end{aligned}\right.
\end{gathered}
$$

From these the following important properties may be inferred:

(i) At short range the GBE interaction is attractive in the symmetric FS pairs and repulsive in the antisymmetric ones.

(ii) Among the FS-symmetrical pairs, the flavor antisymmetric pairs experience a much larger attractive interaction than the flavor-symmetric ones, and among the FSantisymmetric pairs the strength of the repulsion in flavor-antisymmetric pairs is considerably weaker than in the symmetric ones. 
Given these properties we conclude, that with the given flavor symmetry, the more symmetrical the FS Young pattern is for a baryon - the more attractive contribution at short range comes from the GBE. For two identical flavor-spin Young patterns $[f]_{\mathrm{FS}}$ the attractive contribution at short range is larger for the more antisymmetrical flavor Young pattern $[f]_{\mathrm{F}}$.

The flavor-spin symmetry and the sign of the GBE interaction at short range allow to understand the structure of the low-lying baryon spectrum.

For the octet states $\mathrm{N}, \Lambda, \Sigma, \Xi(N=0$ shell, $N$ is the number of harmonic oscillator excitations in a 3-quark state) as well as for their first radial excitations of positive parity $\mathrm{N}(1440), \Lambda(1600), \Sigma(1660), \Xi(?)(N=2$ shell $)$ the flavor and spin symmetries are $[3]_{\mathrm{FS}}[21]_{\mathrm{F}}[21]_{\mathrm{S}}$, and the contribution of the Hamiltonian (5) is $-14 C_{\chi}$. For the decuplet states $\Delta, \Sigma(1385), \Xi(1530), \Omega(N=0$ shell $)$ the flavor and spin symmetries, as well as the corresponding matrix element, are $[3]_{\mathrm{FS}}[3]_{\mathrm{F}}[3]_{\mathrm{S}}$ and $-4 C_{\chi}$, respectively. The negative parity excitations $(N=1$ shell) in the $\mathrm{N}, \Lambda$ and $\Sigma$ spectra $(\mathrm{N}(1535)-\mathrm{N}(1520), \Lambda(1670)$ - $\Lambda(1690)$ and $\Sigma(1750)-\Sigma(?))$ are described by the $[21]_{\mathrm{FS}}[21]_{\mathrm{F}}[21]_{\mathrm{S}}$ symmetries, and the contribution of the interaction (5) in this case is $-2 C_{\chi}$. The first negative parity excitation in the $\Lambda$ spectrum $(N=1$ shell $) \Lambda(1405)-\Lambda(1520)$ is flavor singlet $[21]_{\mathrm{FS}}[111]_{\mathrm{F}}[21]_{\mathrm{S}}$, and, in this case, the corresponding matrix element is $-8 C_{\chi}$. The latter state is unique and is absent in other spectra due to its flavor-singlet nature.

These matrix elements alone suffice to prove that the ordering of the lowest positive and negative parity states in the baryon spectrum will be correctly predicted by the chiral boson exchange interaction (5). The constant $C_{\chi}$ may be determined from the $\mathrm{N}-\Delta$ splitting to be $29.3 \mathrm{MeV}$. The oscillator parameter $\hbar \omega$, which characterizes the effective confining interaction, may be determined as one half of the mass differences between the first excited $\frac{1}{2}^{+}$states and the ground states of the baryons, which have the same flavor-spin, flavor and spin symmetries (e.g. N(1440) - N, $\Lambda(1600)-\Lambda, \Sigma(1660)-$ $\Sigma)$, to be $\hbar \omega \simeq 250 \mathrm{MeV}$. Thus the two free parameters of this simple model are fixed and we can make now predictions.

In the $\mathrm{N}$ and $\Sigma$ sectors the mass difference between the lowest excited $\frac{1}{2}^{+}$states $(\mathrm{N}(1440), \Lambda(1600)$, and $\Sigma(1660))$ and $\frac{1}{2}^{-}{ }^{-} \frac{3}{2}^{-}$negative parity pairs $(\mathrm{N}(1535)-\mathrm{N}(1520)$, $\Lambda(1670)-\Lambda(1690)$, and $\Sigma(1750)-\Sigma(?)$, respectively) will then be

$\mathrm{N}, \Lambda, \Sigma: \quad m\left(\frac{1}{2}^{+}\right)-m\left(\frac{1}{2}^{-}-\frac{3}{2}^{-}\right)=250 \mathrm{MeV}-C_{\chi}(14-2)=-102 \mathrm{MeV}$,

whereas for the lowest states in the $\Lambda$ system $(\Lambda(1600), \Lambda(1405)-\Lambda(1520))$ it should be

$\Lambda: \quad m\left(\frac{1}{2}^{+}\right)-m\left(\frac{1}{2}^{-}-\frac{3}{2}^{-}\right)=250 \mathrm{MeV}-C_{\chi}(14-8)=74 \mathrm{MeV}$

This simple example shows how the GBE interaction provides different ordering of the lowest positive and negative parity excited states in the spectra of the nucleon and the $\Lambda$-hyperon. This is a direct consequence of the symmetry properties of the bosonexchange interaction discussed at the beginning of this section. Namely, the $[3]_{\text {FS }}$ state in the $\mathrm{N}(1440) \Lambda(1600)$ and $\Sigma(1660)$ positive parity resonances from the $N=2$ band feels a much stronger attractive interaction than the mixed symmetry state $[21]_{\mathrm{FS}}$ in the $\mathrm{N}(1535)-\mathrm{N}(1520), \Lambda(1670)-\Lambda(1690)$ and $\Sigma(1750)-\Sigma(?)$ resonances of negative parity 
$(N=1$ shell). Consequently the masses of the positive parity states $\mathrm{N}(1440), \Lambda(1600)$ and $\Sigma(1660)$ are shifted down relative to the other ones, which explains the reversal of the otherwise expected "normal ordering". The situation is different for $\Lambda(1405)-\Lambda(1520)$ and $\Lambda(1600)$, as the flavor state of $\Lambda(1405)-\Lambda(1520)$ is totally antisymmetric. Because of this the $\Lambda(1405)-\Lambda(1520)$ gains an attractive energy, which is comparable to that of the $\Lambda(1600)$, and thus the ordering suggested by the confining oscillator interaction is maintained.

\section{Semirelativistic Chiral Constituent Quark Model}

In the semirelativistic chiral constituent quark model [8,9] the dynamical part of the Hamiltonian consists of linear pairwise confining interaction and the GBE interaction, which includes both the short-range part (parametrized phenomenologically) and the long-range Yukawa tail. In the large $N_{C}$ limit the axial anomaly vanishes [10] and the $\eta^{\prime}$ (the flavor singlet) becomes the ninth Goldstone boson of $U(3)_{R} \times U(3)_{L}$ chiral symmetry [11. Thus, the $\eta^{\prime}$ should be also taken into account, but with coupling constant different as compared to octet Goldstone bosons. The latter coupling constant can be fixed from the known pion-nucleon coupling constant.

The kinetic-energy operator is taken in relativistic form, $\left.H_{0}=\sum_{i=1}^{3} \sqrt{(} p_{i}^{2}+m_{i}^{2}\right)$. The semirelativistic three-quark Hamiltonian was solved along the stochastical variational method [12] in momentum space. For the whole Q-Q potential the model involves a total of 5 free parameters whose numerical values are determined from the fit to all 34 confirmed low-lying states [8,9]. In fig. 1 we present the ground states as well as low-lying excited states in $N, \Delta, \Lambda, \Sigma, \Xi$, and $\Omega$ spectra. From the results of fig. 1 it becomes evident that within the chiral constituent quark model a unified description of both nonstrange and strange baryon spectra is achieved in good agreement with phenomenology.

It is instructive to learn how the GBE interaction affects the energy levels when it is switched on and its strength is gradually increased (Fig. 2). Starting out from the case with confinement only, one observes that the degeneracy of states is removed and the inversion of ordering of positive and negative parity states is achieved in the $N$ spectrum, as well as for some states in the $\Lambda$ spectrum, while the ordering of the lowest positivenegative parity states is opposite in $N$ and $\Lambda$ spectra. The reason for this behaviour is the flavor-spin structure and sign of the short-range part of GBE, discussed in the previous section.

In the semirelativistic model we find that the confinement strength should be consistent with Regge slopes and the string tension extracted in lattice QCD. This is comfortable improvement over nonrelativistic model [13] where a much smaller value of the confinement strength must be chosen in order to reproduce the low-lying spectrum.

It is clear that the Fock components $Q Q Q \pi, Q Q Q K, Q Q Q \eta$, and $Q Q Q \eta^{\prime}$ (including meson continuum) cannot be completely integrated out in favour of the meson-exchange $Q-Q$ potentials for some states above or near the corresponding meson thresholds. Such a component in addition to the main one $Q Q Q$ could explain e.g. an exceptionally big splitting of the flavor singlet states $\Lambda(1405)-\Lambda(1520)$, since the $\Lambda(1405)$ lies below the $\bar{K} N$ threshold and can be presented as $\bar{K} N$ bound system [14]. Note, that in the case of the present approach this old idea is completely natural and does not contradict a flavor- 

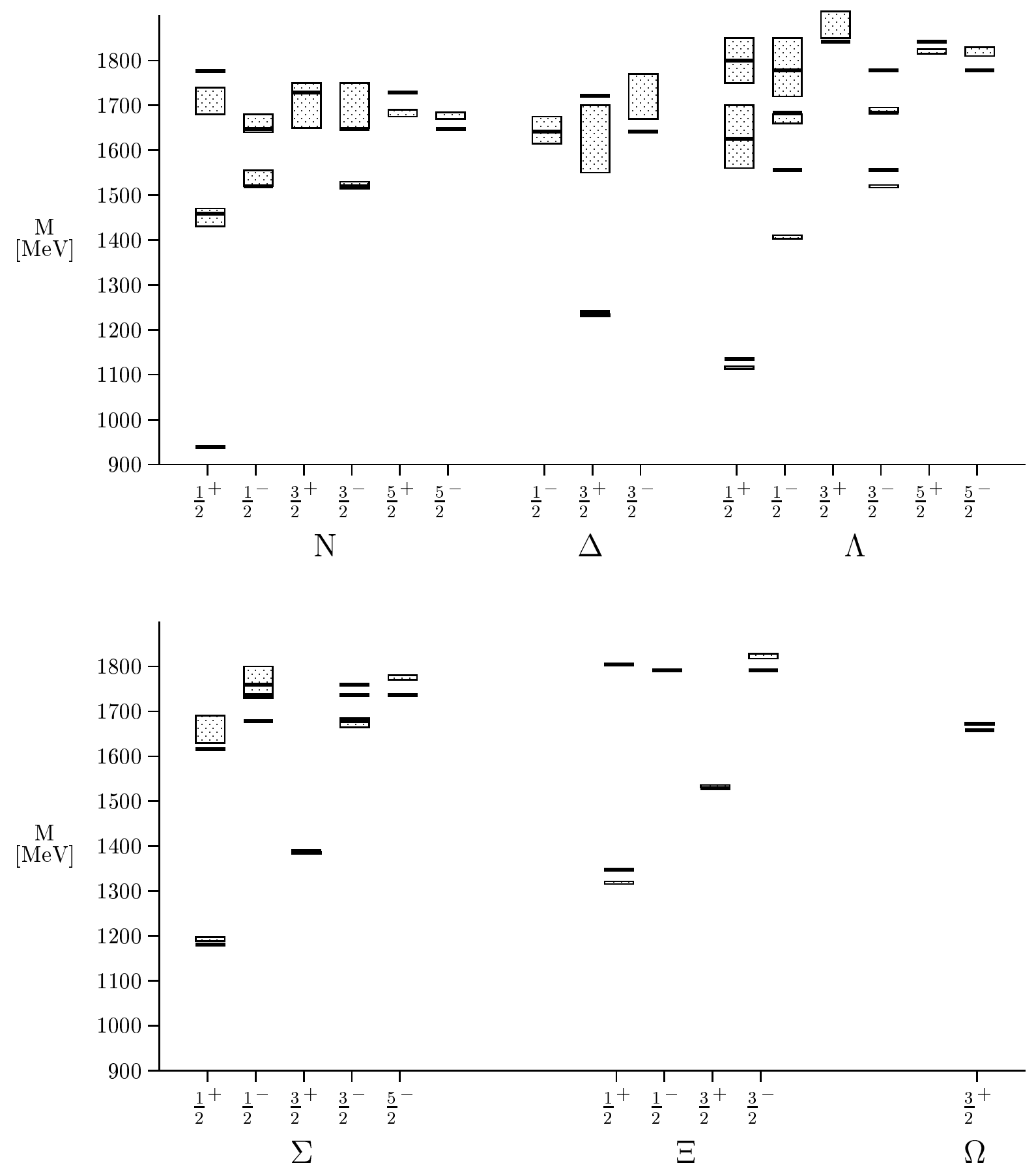

Figure 1. Energy levels of the lowest light and strange baryon states (below $1850 \mathrm{MeV}$ ) with total angular momentum and parity $J^{P}$. The shadowed boxes represent the experimental values with their uncertainties. 

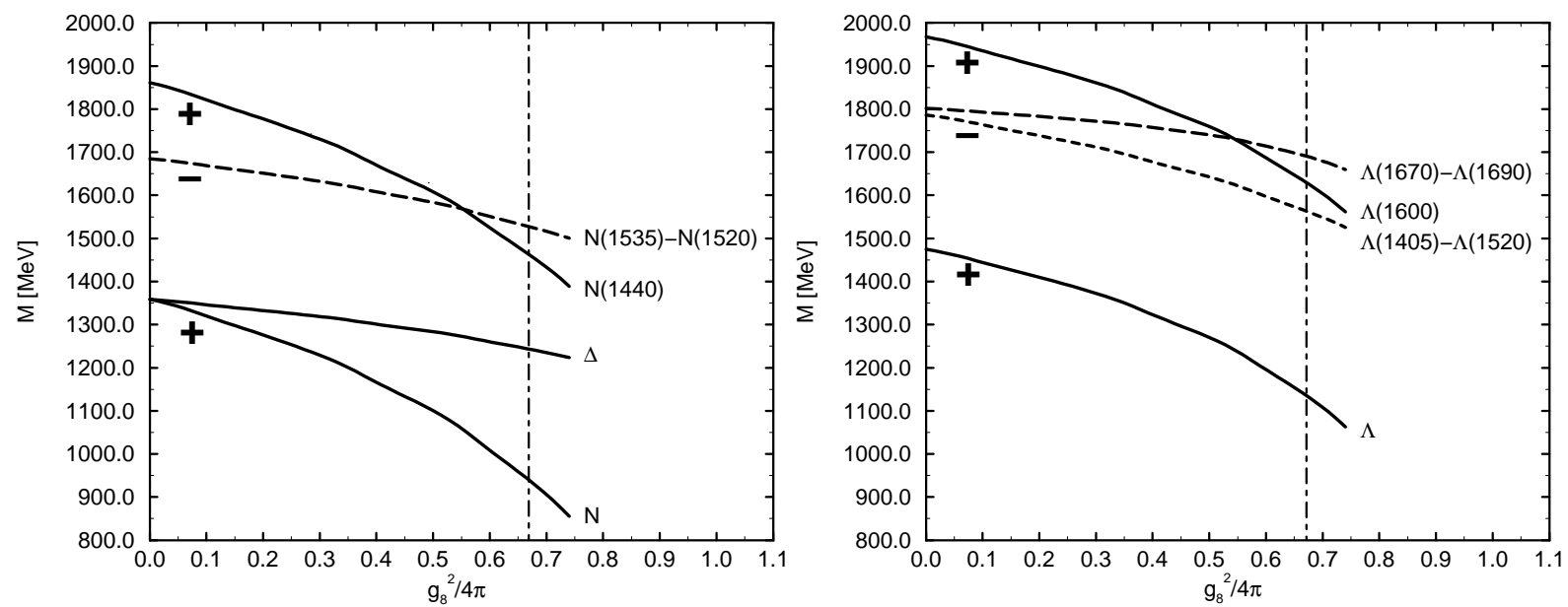

Figure 2. Level shifts of some lowest baryons as a function of the strength of the GBE. Solid and dashed lines correspond to positive- and negative-parity states, respectively.

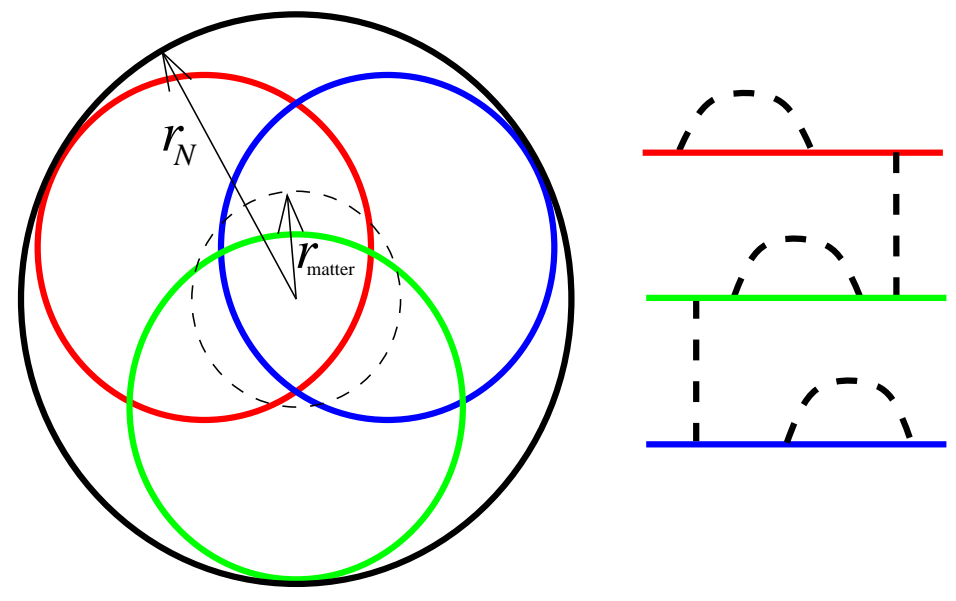

Figure 3. Nucleon as it is seen in the low-energy and low-resolution regime. 
singlet $Q Q Q$ nature of $\Lambda(1405)$, while it would be in conflict with naive constituent quark model where no room for mesons in baryons. An admixture of such components will be important in order to understand strong decays of some excited states. While technically inclusion of such components in addition to the main one $Q Q Q$ in a coupled-channel approach is rather difficult task, it should be considered as one of the most important future directions.

What is an intuitive picture of the nucleon in the low-energy regime? Nucleon consists of 3 constituent quarks which are very big objects due to their meson clouds (see fig. 3). These constituent quarks are all the time in strong overlap inside the nucleon. That is why the short-range part of GBE interaction (which is represented by the contact term in the oversimplified representation (田) is so crucially important inside baryons. When constituent quarks are well separated and there is a phase space for pion propagation, the long-range Yukawa tail of GBE interaction as well as correlated two-pion exchanges become very important. It is these parts of meson exchange which produce the necessary long- and intermediate-range attraction in two-nucleon system.

\section{The Nucleon-Nucleon Interaction in a Chiral Constituent Quark Model}

So far, all studies of the short-range $N N$ interaction within the constituent quark model were based on the one-gluon exchange interaction between quarks. They explained the short-range repulsion in the $N N$ system as due to the colour-magnetic part of OGE combined with quark interchanges between $3 \mathrm{Q}$ clusters [7]. It has been shown, however, that there is practically no room for colour-magnetic interaction in light baryon spectroscopy and any appreciable amount of colour-magnetic interaction, in addition to GBE, destroys the spectrum [8]. This conclusion is confirmed by recent lattice QCD calculations [15]. If so, the question arises which interquark interaction is responsible for the short-range $N N$ repulsion. Below I show that the same short-range part of GBE which causes $N-\Delta$ splitting and produces good baryon spectra, also induces a short-range repulsion in $N N$ system when the latter is treated as $6 \mathrm{Q}$ system [16.

In order to have a qualitative insight into the short-range $N N$ interaction it is convenient to use an adiabatic Born-Oppenheimer approximation for the internucleon potential:

$V_{N N}(R)=<H>_{R}-<H>_{\infty}$,

where $\vec{R}$ is a collective coordinate which is the separation distance between the two $s^{3}$ nucleons, $<H>_{R}$ is the lowest expectation value of the 6Q Hamiltonian at fixed $R$, and $\left\langle H>_{\infty}\right.$ is mass of two well-separated nucleons $\left(2 m_{N}\right)$ calculated with the same Hamiltonian.

It is well known that when the separation $R$ approaches 0 , then for both ${ }^{3} S_{1}$ and ${ }^{1} S_{0}$ partial waves in the $N N$ system only two types of $6 \mathrm{Q}$ configurations survive [17]: $\mid s^{6}[6]_{O}>$ and $\mid s^{4} p^{2}[42]_{O}>$, where $[f]_{O}$ is Young diagram, describing spatial permutational symmetry in $6 \mathrm{Q}$ system. There are a few different flavor-spin symmetries, compatible with spatial symmetries above: $[6]_{O}[33]_{F S},[42]_{O}[33]_{F S},[42]_{O}[51]_{F S},[42]_{O}[411]_{F S},[42]_{O}[321]_{F S}$, and $[42]_{O}[2211]_{F S}$. Thus, in order to evaluate the $N N$ interaction at zero separation between nucleons (i.e. their short-range interaction) it is necessary to diagonalize a $6 Q$ Hamiltonian in the basis above and use the procedure (9). We adjust parameters of the 
"GBE interaction" and "confinement" in a nonrelativistic 3-body calculation to reproduce low-lying $N$ and $\Delta$ spectrum [13]. Clearly, that the nonrelativistic parametrization is only effective (relative to parameters exctracted from the semirelativistic fit). Since we are interested at the moment only in qualitative effects, related to flavor-spin structure and sign of short-range GBE interaction, such an approach can be considered as reasonable.

From the adiabatic Born-Oppenheimer approximation (9) we find that $V_{N N}(R=0)$ is highly repulsive in both ${ }^{3} S_{1}$ and ${ }^{1} S_{0}$ partial waves, with the core being of order 1 $\mathrm{GeV}$. This repulsion implies a strong suppression of the $N N$ wave function in the nucleon overlap region.

Due to the specific flavor-spin symmetry of GBE the configuration $[42]_{O}[51]_{F S}$ becomes highly dominant among other possible $6 \mathrm{Q}$ configurations at zero separation between nucleons (however, the "energy" of this configuration is much higher than the energy of two well-separated nucleons). The symmetry structure of this dominant configuration induces "an additional" effective repulsion, related to the "Pauli forbidden state" in this case [18]. As a result, the s-wave $N N$ relative motion wave function has a node at short range. The existence of a strong repulsion, related to the energy ballance in the adiabatic approximation, suggests, however, that the amplitude of the oscillating $N N$ wave function at short range will be strongly suppressed.

Thus, within the chiral constituent quark model one has all the necessary ingredients to understand microscopically the $N N$ interaction. There appears strong short-range repulsion from the same short-range part of GBE which also produces hyperfine splittings in baryon spectroscopy. The long- and intermediate-range attraction in the $N N$ system is automatically implied by the Yukawa part of pion-exchange and correlated two-pion exchanges between quarks belonging to different nucleons. With this first encouraging result, it might be worthwhile to perform a more elaborate calculation of $N N$ and other baryon-baryon systems within the present framework.

\section{Does H-particle exist?}

It is also interesting to see what will happen at short range in other baryon-baryon systems. In particular, assuming that the colour-magnetic interaction is a main reason for hyperfine splittings in baryons, Jaffe has predicted that there should be well bound dibaryon in $\Lambda \Lambda$ system with $J^{P}=0^{+}$, called H-particle [19]. In this specific case the colour-magnetic interaction in 6q system becomes the most attractive and implies an existence of well-bound dibaryon, stable against $\Lambda \Lambda$ strong decay, since it should be well below this threshold. This H-particle should be compact object in contrast to moleculelike state - deuteron.

It turns out, however, that within our approach there is very strong repulsion at short range in $\Lambda \Lambda$ system with $J^{P}=0^{+}$, coming from the same short-range part of GBE, which induces hyperfine splittings and short-range $N N$ repulsion. This short-range repulsion in $\Lambda \Lambda$ system can be obtained in a similar way [20] to what has been done for $N N$ system in the previous section. To be more specific, the lowest possible 6Q configuration in this case is $\mid s^{6}[6]_{O}[33]_{F S}>$, which lies about $1 \mathrm{GeV}$ above the $\Lambda \Lambda$ threshold. It then suggests, that in our approach to baryon structure the compact H-particle should not exist.

However, there is an attraction in $\Lambda \Lambda$ system at intermediate and long distances which 
has the same nature like in two-nucleon system. The $\Lambda \Lambda$ system with $J^{P}=0^{+}$is similar in this sense to ${ }^{1} S_{0}$ NN partial wave, where the long- and intermediate-range attraction is not enough to bind two-nucleon system.

An extensive experimental search for H-particle for 20 years has showed no positive results [21].

\section{Acknowledgement}

It is my pleasure to thank D.O.Riska, Z.Papp, W.Plessas, K.Varga, R. Wagenbrunn,

Fl. Stancu, and S. Pepin, in collaboration with whom different results discussed in this talk have been obtained.

\section{REFERENCES}

1. M. Gell-Mann and M. Levy, Nuovo Cim. 16 (1960) 705.

2. Y. Nambu and G. Jona-Lasinio, Phys. Rev. 122 (1961) 345; S. P. Klevansky, Rev. Mod. Phys. 64 (1992) 649.

3. C. G. Callan, R. Dashen, and D. J. Gross, Phys. Rev. D17 (1978) 2717; D. I. Diakonov and V. Yu. Petrov, Nucl. Phys. B272 (1986) 457.

4. S. Weinberg, Physica 96 A (1979) 327.

5. A. Manohar and H. Georgi, Nucl. Phys. B234 (1984) 189.

6. L. Ya. Glozman and D. O. Riska, Physics Reports 268 (1996) 263.

7. M. Oka and K. Yazaki, Int. Rev. Nuc. Phys., vol. 1 (Quarks and Nuclei, ed. W. Weise), World Scientific, Singapore, p. 490 (1984).

8. L. Ya. Glozman, Z. Papp, W. Plessas, K. Varga, and R. Wagenbrunn, nuclth/9705011.

9. L. Ya. Glozman, W. Plessas, K. Varga, R. Wagenbrunn, in preparation.

10. E. Witten, Nucl. Phys. B156 (1979) 269.

11. S. Coleman and E. Witten, Phys. Rev. Lett. 45 (1980) 100.

12. K. Varga and Y. Suzuki, Phys. Rev. C52 (1995) 2885; K. Varga, Y. Ohbayasi, and Y. Suzuki, Phys. Lett. B396 (1997) 1.

13. L. Ya. Glozman, Z. Papp, and W. Plessas, Phys. Lett. B381 (1996) 311.

14. R. H. Dalitz and A. Deloff, J. Phys. G 17 (1991) 289 (and references therein).

15. K. F. Liu and S. J. Dong - paper in preparation (for a preliminary result see heplat/9411067).

16. Fl. Stancu, S. Pepin, and L. Ya. Glozman, nucl-th/9705030.

17. M. Harvey, Nucl. Phys. A352 (1981) 301.

18. V. G. Neudatchin, Yu. F. Smirnov, and R. Tamagaki, Progr. Theor. Phys. 58 (1977) 1072.

19. R. L. Jaffe, Phys. Rev. Lett. 38 (1977) 195 ; E: 381617.

20. Fl. Stancu, S. Pepin, and L. Ya. Glozman, in preparation.

21. R. W. Stolzer et al, Phys. Rev. Lett. 78 (1997) 3646 ; see R. E. Chrien's talk. 